\title{
Environmental risk factors for canine atopic dermatitis: A retrospective large-scale study in Labrador and Golden retrievers
}

\author{
Naomi D. Harvey *, Steven C. Shaw * †, Peter J. Craigon *, Sarah C. Blott *, and \\ Gary C.W. England * \\ * School of Veterinary Medicine and Science, The University of Nottingham, Sutton \\ Bonington, Leicestershire, LE12 5RD, United Kingdom \\ † UK VetDerm, 16 Talbot Street, Whitwick, Leicestershire, LE67 5AW, United \\ Kingdom
}

Correspondence: Naomi D. Harvey

E-mail: naomi.harvey@nottingham.ac.uk

Conflicts of interest: The authors declare no conflicts of interest.

Sources of funding: This work was funded by the Dogs Trust Canine Welfare Grant scheme.

This study was presented in part at the UK Companion Animal Genetic Health Conference held at the Royal (Dick) School of Veterinary Studies and Roslin Institute on May $14-15^{\text {th }}, 2018$.

Short running title: Risk factors for canine atopic dermatitis

\section{Abstract}

Background - canine atopic dermatitis (CAD) is one the most common and distressing skin disorders seen in dogs. It is characterised by dysfunction in the skin barrier, with a complex pathogenesis combining both genetic and environmental factors.

Objectives - to evaluate associations between environmental factors and case-control status in two closely related, at-risk breeds, the Labrador and Golden retriever.

Animals - 2,445 pet dogs, of which 793 were classed as cases (575 Labradors and 218 Golden retrievers) and 1,652 as controls (1,120 Labradors and 532 Golden retrievers).

Methods - case-control status was assigned based upon owner response to a standardised validated questionnaire. Retrospective data on rearing environment was collected via additional questions. Univariate and multivariate logistic regressions were utilised to evaluate associations between environmental factors and case-control status.

Results - risk factors included being reared in an urban environment (not living currently in an urban environment), being male, being neutered, receiving flea control, and being allowed on upholstered furniture. Protective factors included living with other 
dogs (not cats) and walking in woodlands, fields or beaches. Additionally, amongst Labradors, chocolate coated dogs were at greater risk of having CAD than black or yellow coated dogs.

Conclusions and clinical importance - This study is the largest of its kind to date to investigate the role of the environment in canine atopic dermatitis. Although precise triggers are unclear, this study contributes to those of earlier studies to highlight the protective role of a rural environment and highlights some novel associations with disease development.

Keywords: dog; atopic dermatitis; epidemiology; allergy; risk factors

\section{Introduction}

Canine atopic dermatitis (CAD) is a genetically mediated disease with heritability estimated to be 0.47 in Labradors and Golden retrievers, ${ }^{1}$ consistent with estimates of human atopic dermatitis heritability, which ranges between $0.43^{2}$ and $0.50 .{ }^{3}$ This suggests that approximately $50 \%$ of the variability in the pathogenesis of $C A D$ is determined by inherited genetics, with $50 \%$ determined by environmental factors.

Whilst the pathogenesis of atopic dermatitis is mediated by defects in the skin barrier and immune dysregulation, ${ }^{4}$ certain exogenous factors have also been implicated including diet, house dust mites (HDM), infections, allergen exposure (or lack thereof) and urban living. ${ }^{5-7}$ With regards to protective factors, probiotics given to mothers and infants have been found to be protective in at-risk human children, 8 whilst in dogs, feeding a home-made diet to lactating mothers was protective in a case-control study of three high-risk dog breeds. ${ }^{9}$ Rural living and living with a dog have also been highlighted as protective for both human children ${ }^{10-12}$ and dogs. ${ }^{13,14}$ Whilst house dust mites act as an allergen in atopic dermatitis, it has been disputed whether naturally occurring levels could play a role in the pathogenesis of the disease ${ }^{6,15,16}$ with one study finding no association with HDM levels in the house and CAD risk ${ }^{17}$. However, exposure to endotoxins (pro-inflammatory highly toxic molecules found in cell walls of Gram-negative bacteria) early in development have been shown to have an inverse relationship with $A D$ risk in both children ${ }^{18}$ and dogs, 17 which may help to explain the protective effects of rural living and living with other dogs. Considering the large role that environment plays in the pathogenesis of this complex disease, the data on the role of environment in CAD risk is surprisingly scarce with only four case-control studies published to date which investigate environmental risk factors for CAD. 9,13,14,17

Prevalence estimates for CAD range from 2.6 to $15 \%{ }^{19,20}$ with differences likely due to variation in study population, methodology and diagnostic criteria to name a few. However, all estimates of CAD prevalence are likely to be underestimates due to factors highlighted by Hillier \& Griffin. ${ }^{20}$ Although some sources have speculated at an increase in prevalence of CAD, any potential increases in prevalence could be due instead to improved diagnosis and recognition of this complex disease, or alternatively to a rise in popularity of high-risk breeds, as opposed to any rise in environmental contributing factors. All we can be certain of is that a significant 
proportion of the world's dog population develop cAD, often at a detriment to their long-term welfare and potentially also the relationship with their human owner. ${ }^{21}$ In conjunction with the strong similarities between $C A D$ and human- $A D,{ }^{22}$ these factors make $C A D$ an important disease on which to focus veterinary research efforts.

A questionnaire-based tool, the canine atopic dermatitis research questionnaire (cAD-RQ), was recently developed for collecting epidemiological information on CAD and associated skin diseases in dogs, directly from their owners. ${ }^{23}$ Such a tool offers the ability to collect detailed information on skin conditions in dogs on a larger scale than has ever been seen before. By collecting information (much of it retrospective) directly from dog owners, experimental control over factors such as the rigour of diagnosis/screening is lost, and the results are open to recall errors. However, this is balanced by the power of a large data set, allowing for population-level averages to be made, reducing the impact of such errors. Questionnaires have been successfully developed and validated for the purpose of collecting epidemiological data (e.g. ${ }^{24,25}$ ) and are a valuable addition to the researcher's tool kit.

The aim of this study was to utilise data collected by the CAD-RQ to evaluate the demographic and environmental risk factors for CAD in a large sample of dogs, predominantly from the UK. The present study was conducted as part of a larger initiative, which aims to investigate the role of genetic and environmental factors in the development of CAD, focussing on two closely related breeds of dog that are popular in the UK and worldwide; the Labrador and Golden retriever. Whilst these breeds are rarely considered to be at high risk for $\mathrm{CAD}$, Labradors appear in the top 15 breeds with insurance claims for atopic dermatitis in insurance companies in Sweden, ${ }^{14}$ and according to data released by UK insurance company PetPlan based on 2016 claims, Labradors were in the top three dog breeds to claim for this condition. ${ }^{26}$ Golden retrievers, a close genetic relative of the Labrador, have been shown to have an increased prevalence for other skin conditions such as acute moist dermatitis, ${ }^{19}$ and as with Labradors, they are well suited to epidemiological studies due to their large numbers and popularity.

\section{Methods}

This study was reviewed and approved by the University of Nottingham's School of Veterinary Medicine and Science clinical ethical review panel prior to data collection commencement (identification reference 1979,170217).

The data analysed here was collected as part of a retrospective cross-sectional study in conjunction with the canine atopic dermatitis research questionnaire (CAD$\mathrm{RQ}$ ), which was completed by owners of purebred Labradors and Golden retrievers. Full details of the $C A D-R Q$, including distribution methods and demographic details of the entire population can be found in. ${ }^{23}$ Enrolment for the study was open for a 4month period in 2017, and it was advertised via the Kennel Club, press releases, and social media. Out of a pool of 4,111 useable responses, cases were identified as dogs whose owners reported them to have received a veterinary diagnosis for canine atopic dermatitis, and controls were dogs whose owners reported 'No skin related 
diagnosis', no 'Current or past areas of abnormal skin (i.e. red, patchy, hairless, rough, swollen or discoloured)' and no 'Current or past signs of abnormal itchiness (frequent and recurrent rubbing, licking, chewing or scratching of the same areas)'. Given the classification of a case as a dog which has received a diagnosis of CAD (from either a general practitioner or specialist), the population studied here represents the heterogenous population of dogs in first opinion practice that are diagnosed with (and subsequently treated for) CAD. In this sense we are referring to broad-spectrum CAD, which includes that associated with food allergies. Dogs whose owners reported them to have received a diagnosis specifically of food allergies and not environmental allergies were excluded, as were dogs whose owners reported them to only have received a diagnosis of flea allergic dermatitis. Validation of this classification of a case as broad-spectrum CAD is provided in the original manuscript. ${ }^{23}$ In total, data from 2,445 dogs consisting of 1,695 Labrador retrievers (69\%) and 750 Golden retrievers (31\%) were used for this analysis, of which 793 were classed as Cases (575 Labradors and 218 Golden retrievers) and 1,652 Controls (1,120 Labradors and 532 Golden retrievers).

Alongside the $\mathrm{CAD}-\mathrm{RQ}$, owners were asked a further 19 questions about the dogs' home environment, early years and current lifestyle (S. Table 1). Where 'I don't know' was selected, the question was treated as missing data. Dog breed, sex and (for Labradors only) coat colour, was recorded during registration for the project, and whether the dog received flea and/or tick treatment was ascertained in response to the $\mathrm{CAD}-\mathrm{RQ}$ as an additional environmental variable.

Owners were asked to select the category that best described where their dog was born [Rural (countryside, village, or edge of town bordering countryside); Urban (town or city); Other; I don't know]. Additionally, where an owner provided a postcode for the location of the dog's current home, their location was classified into a 1-10 rural/urban scale as a measure of how rural/urban the dogs' current environment was. To create the scale, data for the Rural and Urban classification for England and Wales 2011 for Output Areas was sourced from the Office for National Statistics Open Geography Portal (https://ons. maps.arcgis.com/home/item.html?id=3ce248e9651f4dc094f84a4c5de18 655) and data for the Scottish Government Rural Urban Classification 2016 was downloaded from the Scottish government website (http://www.gov.scot/Publications/2018/03/6040/downloads). As the two Rural Urban Classifications contained a different number of categories (10 in England and Wales and 8 in Scotland) they were both ordered from most urban to most rural and the Scottish scale was then divided by 8 and multiplied by 10 , then rounded to the nearest integer to become equivalent to the England and Wales 1-10 scale.

\section{Statistical analysis}

All questions with nominal answers were dummy coded in Excel so that 1 meant a 'yes' to the given answer and 0 meant 'no'. Written answers to 'Other' selections were evaluated and categorised to the corresponding answer option where appropriate, and all remaining 'Other' answers that could not be grouped were treated as missing data. Data was analysed in SPSS v.22 (SPSS Inc., Chicago, IL). 
Following descriptive analysis checks, data were analysed using binary logistic regressions, with case or control as the outcome. Each question first underwent a separate univariate analysis, and those that were significant to $p<0.15$ were retained for further testing. ${ }^{27}$ In order to avoid multicollinearity, all variables retained for further testing that had potential associations with each other were evaluated using $\mathrm{Chi}^{2}$ tests. Where significant associations $(\mathrm{p}<0.05)$ were found between questions, the one with the largest effect size (Wald value) was retained for multivariate analysis. Where dummy coded answer options to the same question were positively associated to each other they were combined into a single answer category and iteratively re-tested against remaining answers within that question until no further combinations could be made. For example, question 12 provides six answer options for where dogs are walked. This analysis revealed strong positive correlations between dogs walked in residential roads and parks/grassed areas, so these were combined into one answer option of being walked residential roads $O R$ parks/grassed areas, and then retested against the remaining variables until no further groups could be formed.

All variables that remained after these procedures were entered into an automatic forwards multivariate regression model, followed by a backwards regression to confirm the model structure. Breed and sex were forced into the final model to control for their potential confounding effects.

\section{Results}

Two questions could not be analysed: question 5 , regarding what the dam was fed during pregnancy was answered by too few Cases (only 26 Cases had data on this, compared to 117 Controls). Question 9 had no variation, as all dogs had daily access to a garden/yard.

The results of the univariate analysis can be seen in Table 1 . In total, 16 potential risk factors were revealed plus 9 potentially protective factors. Nineteen variables were retained for further analysis, of which 15 were tested for collinearity using $\mathrm{Chi}^{2}$ tests (Table 1). Five of the 15 variables entered for $\mathrm{Chi}^{2}$ analysis were retained for entry into the multivariate model: Born rural; Lives with other dogs; Walked in fields, woods or beaches; Sleeps in a crate bed, and Spends time on upholstery (see S. Table 2 for $\mathrm{Chi}^{2}$ results on question answer groupings). A total of 9 variables were entered into the multivariate model.

It is worth noting that although not included in the multivariate model, coat colour in Labrador retrievers was significantly associated with risk of having a diagnosis of CAD. Chocolate Labradors were $1.94 x$ more likely to have CAD than Black coated Labradors $(\mathrm{OR}=1.94, \mathrm{p}<0.001$, Wald $=20.86$ ), whilst there was no significant difference between Black and Yellow coated Labradors $(p=0.131$, Wald $=2.28)$.

\section{Final multivariate model}

Seven significant factors were retained in the final model, plus breed, which was not significantly associated with cAD risk (Table 2). Of these seven, four were risk 
factors for the disease (each associated with mildly increased risk) and three were protective factors. With regard to risk factors, males $(\mathrm{p}<0.001, \mathrm{OR}=1.62)$ and neutered dogs of both sexes $(p<0.001, O R=1.87)$ were more likely to be diagnosed with $C A D$; for males this would have an additive affect with a neutered male being more likely to develop $c A D$ than an intact female. Cases were also more likely to be administered flea/tick treatment $(p<0.001, O R=1.92)$ and more likely to spend time on upholstery $(p=0.001, O R=1.41)$. Whilst these are referred to as 'risk factors' it must be remembered that cause and effect cannot be established, as associations such as mildly increased use of flea/tick treatment amongst cases could be as a result of the dog's diagnosis itself.

Three potentially protective factors remained in the multivariate model, associated with dogs that did not have CAD. These include a reduced risk of developing CAD for dogs born in a rural location $(p=0.013, O R=0.74)$, dogs that live with other dogs $(p=0.001, O R=0.73)$, and dogs that are walked in woodlands, fields or beaches $(p<0.001, O R=0.48)$.

\section{Discussion}

Four previous case-control studies have investigated environmental risk factors for CAD, $9,13,14,17$ whilst a further two recent studies employed questionnaire methods to investigate risk factors for allergic symptoms. ${ }^{22,28}$ For one of the questionnaire studies, approximately $55 \%$ of those classified as cases had a reported veterinary diagnosis of CAD, ${ }^{28}$ and the number of dogs with a diagnosis of CAD in the second is unknown. ${ }^{23}$ Here, we analysed data for 793 dogs with a veterinary diagnosis of CAD and 1,652 carefully defined controls, from two closely related breeds; Labrador and Golden retrievers. Considering the four previously published case-control studies only, the current study represents the largest and most comprehensive study of its kind into the environmental risk factors for canine atopic dermatitis.

Most comparable to the current work was a case-control study focussing on Labrador and Golden retrievers in Switzerland \& Germany. ${ }^{13}$ Meury and colleagues were able to recruit 378 dogs, which underwent clinical diagnoses for CAD as part of the study and whose owners completed a 46-item questionnaire on environmental factors. In the current study, being reared in a rural environment was associated with a reduced risk of developing CAD, which supports previous findings for dogs in Sweden, ${ }^{14}$ Switzerland \& Germany. ${ }^{13}$ However, the environment they currently lived in was not significantly associated with whether they were a case or control, which is in contrast to the results of Hakanen and colleagues who found both dogs and their owners were more likely to have allergies if they lived in an urban environment. ${ }^{23}$ In the UK at least, it seems that the protective effect of rural environments is gained during early life.

Meury and colleagues reported that living with other 'dogs or cats' and walking in woodlands proffered protective effects against CAD. Here, we were able to go further to show that it is living with other dogs, as opposed to cats that offers this protective effect, and that walks in fields or beaches also act as protective factors in addition to 
walking in woodlands. It is possible that combined with the protective effect of being reared somewhere rural, that a more rural lifestyle in general is a protective factor for CAD development, as with human studies. ${ }^{11}$ Interestingly, living with a dog has also been implicated to be protective with regards to development of childhood human atopic dermatitis, potentially due to increased early exposure to a range of allergens. 10,12 Allergies have been reported more often for Finnish dogs that lived in an 'extremely clean' household ${ }^{28}$ and exposure to endotoxins secreted by Gramnegative bacteria have been shown to be inversely related to CAD risk ${ }^{17}$, all of which supports the hygiene hypothesis of allergy risk theory. ${ }^{29}$

Findings unique to the current study include significant risk enhancing effects of being male, of being neutered, use of flea/tick control and being allowed to spend time on upholstered furniture. An additional finding relevant only to Labradors was that dogs with chocolate coloured coats were 1.94x more likely to have a diagnosis of CAD than were black or yellow dogs. This result makes it imperative that Labrador breeding schemes, especially those from chocolate coated lineages, reduce the use of dogs with clinical signs of $C A D$ in breeding for the future welfare of the breed. Whilst previous work has shown an association between CAD risk and having more than $50 \%$ white coat colouring in dogs ${ }^{28}$ we could not confirm this finding here as the breeds utilised in the current study do not commonly have white fur.

Studies from human medicine indicate an impact of sex hormones in the pathogenesis of atopic diseases, with adult females being more likely to suffer from $A D{ }^{30}$. However, previous findings for sex effects on CAD risk have been conflicting and could be partially explained by differences in geographical location or neuter status of the study populations. Many studies where dogs were reported to be predominantly intact have found no sex differences in CAD risk, 1,9,14 (although see ${ }^{31}$ where females were more likely to have positive IgE results in a mixed breed predominantly intact Norwegian sample). Here, the population was predominantly desexed (66\%) and a significant effect was found for neutering increasing the risk of CAD in both sexes. Additionally, males of these breeds were over-represented amongst cases of CAD; a finding supported by Picco and colleagues ${ }^{32}$ from a small sample of Swiss Labradors. Whilst neutering was found to increase risk of CAD, at least in these breeds, neutering can proffer many protective effects against other diseases. ${ }^{33}$ Age at time of neuter was approaching significance at $p<0.1$ in univariate models, suggesting that the timing of spay/castration could impact the risk enhancing effect of neutering on CAD development.

The association between time spent on upholstered furniture and increased risk of $C A D$ could be considered to support previous suggestions that house dust mite exposure could play an important role in the manifestation of CAD. ${ }^{1,34-36}$ Indeed, house dust mite levels have been implicated in the pathogenesis of human atopic diseases. ${ }^{6,15}$ Although management guidelines for dogs diagnosed with $C A D$ already include controlling house dust mite exposure, it may be suitable to suggest that such measures be taken in advance of clinical signs as a preventative measure for any dogs with genetic risk of the disease. Further, the fact that existing cases of CAD were more likely than controls to spend time on upholstery suggests that owners are not enforcing proper management regimens for reducing dust mite exposure in dogs 
with atopic allergies. This is important to know for veterinarians of atopic patients, who can try to reinforce the need for owners to take the appropriate management steps to reduce their dog's allergy load, which should result in improved clinical signs. 36

As with all such retrospective correlational studies it must be remembered that cause and effect cannot be established. Results such as cases being 1.92x more likely to be administered flea control are likely to be associated with the diagnosis itself; due to owners administering flea control as a result of the dog's skin disease itself, since flea control is recommended for dogs with $C A D{ }^{37}$. The same is possible for the places the dogs are walked, with owners of dogs diagnosed with CAD perhaps being less likely to walk them in woods, fields or beaches after diagnosis, due to potential for increased allergen exposure, meaning that control dogs are overrepresented in these areas. Questions of cause and effect can only be answered by prospective cohort studies, which are warranted if we are to better understand the role of environment in the pathogenesis of CAD.

Epidemiological studies of complex diseases such as atopy require large sample sizes in order to detect the small cumulative effects that could be present from environmental factors amongst other existing variables. Thus, a balance is to be struck between small-scale studies that offer more scope for controlling variables and large-scale studies that may be difficult to manage but offer more statistical power. Here, we utilised a validated questionnaire, and defined a case as a dog that had received a veterinary diagnosis of $C A D$. Thus, our definition of CAD was very broad and there is a greater risk that dogs were misdiagnosed. However, this risk is tradedoff with the greater power offered by a large dataset, which should not be overly impacted by low rates of potential misdiagnosis.

This study is the largest of its kind to date to investigate the role of the environment in canine atopic dermatitis. Precise triggers are unclear, but the results presented here contribute to those of earlier studies to highlight the protective role of a rural environment, support recommendations that dogs with CAD (or at risk of CAD) avoid spending time on upholstery and highlight a novel role of neutering in disease development. In terms of disease prevention, future research should investigate the possible genetic mechanisms for coat colour for increasing risk of CAD in chocolate Labradors, and extra care should be taken when breeding from lines that include chocolate colour phenotypes. Care should perhaps also be taken in deciding whether to neuter a male dog of these breeds when it is known that CAD is present in its family history. In terms of patient management, owners should be reminded of the risks posed by allowing their dogs onto upholstery. Longitudinal, cohort-based studies are warranted to further elucidate the role of these specific environmental factors in CAD development.

\section{References}

1. Shaw SC, Wood JLN, Freeman J, et al. Estimation of heritability of atopic dermatitis in Labrador and Golden Retrievers. Am J Vet Res 2004; 65: 1014-1020. 
2. Gerrard JW, Rao DC, Morton NE. A genetic study of immunoglobulin E. Am J Hum Genet 1978; 30: 46-58.

3. Blumenthal MN, Namboodiri K, Mendell N, et al. Genetic transmission of serum IgE levels. Am J Med Genet 1981; 10: 219-228.

4. $\quad$ Bieber T. Atopic Dermatitis. N Engl J Med 2008; 358: 1483-1494.

5. McPherson T. Current Understanding in Pathogenesis of Atopic Dermatitis. Indian J Dermatol 2016; 61: 649-655.

6. Landheer J, Giovannone B, Mattson JD, et al. Epicutaneous application of house dust mite induces thymic stromal lymphopoietin in nonlesional skin of patients with atopic dermatitis. J Allergy Clin Immunol 2013; 132: 1252-1254.

7. Flohr C, Pascoe D, Williams HC. Atopic dermatitis and the 'hygiene hypothesis': Too clean to be true? Br J Dermatol 2005; 152: 202-16.

8. Kukkonen K, Savilahti E, Haahtela T, et al. Probiotics and prebiotic galactooligosaccharides in the prevention of allergic diseases: A randomized, doubleblind, placebo-controlled trial. J Allergy Clin Immunol 2007; 119: 192-198.

9. Nødtvedt A, Bergvall K, Sallander M, et al. A case - control study of risk factors for canine atopic dermatitis among boxer, bullterrier and West Highland white terrier dogs in Sweden. Vet Dermatol 2007; 18: 309-315.

10. Cheema G, Sitarik A, Havstad S, et al. Effect of prenatal dog exposure on eczema development in early and late childhood. Ann Allergy, Asthma Immunol 2017; 119: S14.

11. Ciaccio CE, Portnoy JM. Strategies for primary prevention of atopy in children. Curr Allergy Asthma Rep 2008; 8: 493-9.

12. Biagini Myers JM, Wang N, LeMasters GK, et al. Genetic and environmental risk factors for childhood eczema development and allergic sensitization in the CCAAPS cohort. J Invest Dermatol 2010; 130: 430-7.

13. Meury S, Molitor V, Doherr MG, et al. Role of the environment in the development of canine atopic dermatitis in Labrador and golden retrievers. Vet Dermatol 2011; 22: $327-334$.

14. Nødtvedt A, Egenvall A, Bergvall K, et al. Incidence of and risk factors for atopic dermatitis in a Swedish population of insured dogs. Vet Rec 2006; 159: 241-246.

15. Tupker RA, De Monchy JGR, Coenraads PJ, et al. Induction of atopic dermatitis by inhalation of house dust mite. J Allergy Clin Immunol 1996; 97: 1064-1070.

16. Zur G, Ihrke PJ, White SD, et al. Canine atopic dermatitis: a retrospective study of 266 cases examined at the University of California, Davis, 1992-1998. Part I. Clinical features and allergy testing results. Vet Dermatol 2002; 13: 89-102. 
17. Looringh van Beeck FA, Hoekstra H, Brunekreef B, et al. Inverse association between endotoxin exposure and canine atopic dermatitis. Vet J 2011; 190: 215219.

18. Gehring U, Bolte G, Borte $\mathrm{M}$, et al. Exposure to endotoxin decreases the risk of atopic eczema in infancy: A cohort study. J Allergy Clin Immunol 2001; 108: 847854.

19. Wiles BM, Llewellyn-Zaidi AM, Evans KM, et al. Large-scale survey to estimate the prevalence of disorders for 192 Kennel Club registered breeds. Canine Genet Epidemiol 2017; 4: 1-18.

20. Hillier A, Grif CE. The ACVD task force on canine atopic dermatitis ( I): incidence and prevalence. Vet Immunol Immunopathol 2001; 81: 147-151.

21. Noli C, Minafo G, Galzerano M. Quality of life of dogs with skin diseases and their owners . Part 1 : development and validation of a questionnaire. Vet Dermatol 2011; 22: 335-343.

22. Hakanen E, Lehtimäki J, Salmela E, et al. Urban environment predisposes dogs and their owners to allergic symptoms. Sci Rep 2018; 8: 1585.

23. Harvey ND, Shaw SC, Blott SC, et al. Development and validation of a new standardised data collection tool to aid in the diagnosis of canine skin allergies. Sci Rep 2019; 9: 3039.

24. Roine J, Uusitalo L, Hielm-Björkman A. Validating and reliability testing the descriptive data and three different disease diagnoses of the internet-based DOGRISK questionnaire. BMC Vet Res 2016; 12: 1-7.

25. Pugh CA, Bronsvoort BMDC, Handel IG, et al. Dogslife: A cohort study of Labrador Retrievers in the UK. Prev Vet Med. Epub ahead of print 2015. DOI: 10.1016/j.prevetmed.2015.06.020.

26. Top five health problems for dogs, cats, rabbits and horses | Blue Cross, https://www.bluecross.org.uk/pet-advice/top-five-health-problems-dogs-catsrabbits-and-horses (accessed 4 January 2018).

27. Dohoo I, Martin W, Styhnn H. Logistic regression. In: Pike M (ed) Veterinary Epidemiologic Research. Charlottetown, Canada: Atlantic Veterinary College, 2003.

28. Anturaniemi J, Uusitalo L, Hielm-Bjorkman A. Environmental and phenotyperelated risk factors for owner-reported allergic/atopic skin symptoms and for canine atopic dermatitis verified by veterinarian in a Finnish dog population. PLoS One 2017; 12: 1-17.

29. Okada H, Kuhn C, Feillet H, et al. The 'hygiene hypothesis' for autoimmune and allergic diseases: an update. Clin Exp Immunol 2010; 160: 1-9.

30. Chen W, Mempel M, Schober W, et al. Gender difference, sex hormones, and 
immediate type hypersensitivity reactions. Allergy 2008; 63: 1418-1427.

31. Bjelland AA, Dolva FL, Nødtvedt A, et al. Prevalence of and risk factors for increased serum levels of allergen-specific IgE in a population of Norwegian dogs. Acta Vet Scand 2014; 56: 81.

32. Picco F, Zini E, Nett C, et al. A prospective study on canine atopic dermatitis and food-induced allergic dermatitis in Switzerland. Vet Dermatol 2008; 19: 150-155.

33. Reichler I. Gonadectomy in Cats and Dogs: A Review of Risks and Benefits. Reprod Domest Anim 2009; 44: 29-35.

34. Hill PB, Deboer DJ. The ACVD task force on canine atopic dermatitis (IV): environmental allergens. Vet Immunol Immunopathol 2001; 20: 169-86.

35. Nuttall TJ, Hill PB, Bensignor E, et al. House dust and forage mite allergens and their role in human and canine atopic dermatitis. Vet Dermatol 2006; 17: $223-$ 235.

36. Swinnen C, Vroom M. The clinical effect of environmental control of house dust mites in 60 house dust mite-sensitive dogs. Vet Dermatol 2004; 15: 31-36.

37. Shaw S. A therapeutic approach to allergic pruritus in the dog. In Pract 2013; 35: 24-28.

\section{Supporting Information}

The Supporting Information provided is intended to be made available through the online edition.

S. Table 1: Questions asked alongside the $\mathrm{CAD}-\mathrm{RQ}$. ${ }^{*}$ Indicates those that were only shown depending on the answer to the previous question.

S. Table 2: Results of $\mathrm{Chi}^{2}$ tests for association between related variables, and dummy coded question answers. Variables in group $C$ were from the same question so those with positive relationships were combined into a single variable. If the relationship was negative they were not combined, but the one with the higher Wald value was retained for the MV. For all other groups, the question with the highest Wald value was retained where associations were present. When no significant association occurred, both questions were retained for the multivariate model. 
Table 1. Results of initial univariate binary regressions of question variables compared to the dependent variable "Case or Control" for canine atopic dermatitis $(n=2,445)$ in Labrador and Golden retrievers. Odds ratios, with lower and upper confidence intervals shown for variables significant to $p<0.15$. Outcomes include: retain for multivariate model (Retain $-\mathrm{MV}$ ), retain for collinearity analysis (Retain $-\mathrm{Chi}^{2}$ ), exclude from further analysis because $\mathrm{p}<0.15$ (Exclude), exclude from further analysis because only part of the population answered the question (Exclude ${ }^{\star}$.

\begin{tabular}{|c|c|c|c|c|c|c|c|c|}
\hline Question & Variables & Answer category & $\mathbf{p}$ & Wald & OR & Lower C.I & Upper C.I & Outcome \\
\hline cAD-RQ & Flea/tick control given $(1 / 0)$ & & $<0.001$ & 15.38 & 1.86 & 1.36 & 2.53 & Retain - MV \\
\hline \multirow[t]{3}{*}{ cAD-RQ } & Labrador coat colour (comparator: Black) & & $<0.001$ & 20.86 & & & & Exclude* \\
\hline & & Yellow & 0.131 & 2.28 & 1.21 & 0.95 & 1.55 & \\
\hline & & Chocolate & $<0.001$ & 20.86 & 1.94 & 1.46 & 2.58 & \\
\hline 1 & Neutered $(1 / 0)$ & & $<0.001$ & 45.71 & 1.94 & 1.60 & 2.35 & Retain - MV \\
\hline 2 & Age neutered (months)* & & 0.087 & 2.93 & 0.99 & 0.99 & 1.00 & Exclude* \\
\hline \multirow[t]{3}{*}{3} & Rearing environment (comparator: Indoors) & & 0.418 & 1.74 & & & & Exclude \\
\hline & & Outdoor Kennel & 0.600 & 0.03 & & & & Exclude \\
\hline & & Outdoor shed & 0.238 & 1.40 & & & & Exclude \\
\hline \multirow[t]{6}{*}{4} & Age homed (comparator: 6-8 weeks) & & 0.010 & 15.06 & & & & Re-coded \\
\hline & & 9-11 weeks & 0.653 & 0.20 & & & & Re-coded \\
\hline & & 3-5 months & 0.451 & 0.57 & & & & Re-coded \\
\hline & & 6-12 months & 0.393 & 0.73 & & & & Re-coded \\
\hline & & Older than 1 year & 0.894 & 0.02 & & & & Re-coded \\
\hline & & Bred myself & $<0.001$ & 12.75 & 0.45 & 0.29 & 0.70 & Re-coded \\
\hline 4 - recoded & Bred by owner (Bred myself $=1$, All else $=0$ ) & & $<0.001$ & 13.27 & 0.45 & 0.29 & 0.69 & Retain - MV \\
\hline 7 & Born rural $(1 / 0)$ & & $<0.001$ & 14.65 & 0.64 & 0.51 & 0.80 & Retain - Chi ${ }^{2}$ grp. A \\
\hline \multirow[t]{3}{*}{8} & Dog lives (comparator: Inside) & & $<0.001$ & 11.91 & & & & Re-coded \\
\hline & & Outside & 0.001 & 11.38 & 0.232 & 0.099 & 0.54 & Re-coded \\
\hline & & Mixed & 0.402 & 0.70 & & & & Re-coded \\
\hline 8 - recoded & Dog lives Outside (1/0) & & 0.001 & 11.20 & 0.234 & 0.1 & 0.55 & Retain - Chi ${ }^{2}$ grp. A \\
\hline \multirow[t]{3}{*}{10} & Carpeting (comparator: None) & & 0.015 & 8.46 & & & & Re-coded \\
\hline & & Mixed & 0.004 & 8.46 & 1.36 & 1.11 & 1.67 & Re-coded \\
\hline & & All carpet & 0.131 & 2.28 & & & & Re-coded \\
\hline 10 - recoded & Mixed flooring (Mixed $=1$, All else $=0$ ) & & 0.012 & 6.32 & 1.26 & 1.05 & 1.50 & Yes - MV \\
\hline 11 & Lives with other dogs $(1 / 0)$ & & $<0.001$ & 26.18 & 0.64 & 0.54 & 0.76 & Retain - Chi ${ }^{2}$ grp. B \\
\hline 11 & Lives with cats $(1 / 0)$ & & 0.898 & 0.02 & & & & Exclude \\
\hline 11 & Live with children $(1 / 0)$ & & 0.306 & 1.05 & & & & Exclude \\
\hline 11 & Lives alone $(1 / 0)$ & & 0.009 & 6.87 & 1.29 & 1.07 & 1.55 & Retain - Chi² grp. B \\
\hline 12 & Walked in Woodlands $(1 / 0)$ & & $<0.001$ & 13.88 & 0.71 & 0.60 & 0.85 & Retain - Chi ${ }^{2}$ grp. C \\
\hline 12 & Walked in Fields $(1 / 0)$ & & $<0.001$ & 13.29 & 0.70 & 0.58 & 0.85 & Retain - Chi ${ }^{2}$ grp. C \\
\hline 12 & Walked on Residential roads $(1 / 0)$ & & 0.012 & 6.32 & 1.25 & 1.05 & 1.48 & Retain - Chi $^{2}$ grp. C \\
\hline 12 & Walked in Parks/grassed play area $(1 / 0)$ & & $<0.001$ & 28.32 & 1.59 & 1.34 & 1.89 & Retain - Chi $^{2}$ grp. C \\
\hline
\end{tabular}




\begin{tabular}{|c|c|c|c|c|c|c|c|}
\hline 12 & Walked in Beach/coastal areas $(1 / 0)$ & 0.139 & 2.19 & 0.86 & 0.70 & 1.05 & Retain - Chi ${ }^{2}$ grp. C \\
\hline 13 & Diet type (Dry vs. All else) & 0.191 & 6.11 & & & & Exclude \\
\hline \multirow[t]{3}{*}{15} & Time on Upholstery (comparator: Daily) & $<0.001$ & 18.99 & & & & Re-coded \\
\hline & Sometimes & 0.961 & 0.00 & & & & Re-coded \\
\hline & Never & $<0.001$ & 17.20 & 0.66 & & 0.80 & Re-coded \\
\hline 15 - recoded & Spends time on upholstery $($ Yes + Sometimes $=1$, Never $=0)$ & $<0.001$ & 18.99 & 1.52 & 1.26 & 1.84 & Retain - Chi ${ }^{2}$ grp. D \\
\hline 16 & Sleeps on Dog bed $(1 / 0)$ & 0.738 & 0.11 & & & & Exclude \\
\hline 16 & Sleeps on Human bed $(1 / 0)$ & 0.004 & 8.32 & 1.36 & 1.11 & 1.68 & Retain - Chi ${ }^{2}$ grp. D \\
\hline 16 & Sleeps on Sofa $(1 / 0)$ & 0.011 & 6.52 & 1.29 & 1.06 & 1.56 & Retain - Chi ${ }^{2}$ grp. D \\
\hline 16 & Sleeps on Carpeted floor $(1 / 0)$ & 0.122 & 2.39 & 1.16 & 0.96 & 1.41 & Retain - Chi ${ }^{2}$ grp. D \\
\hline 16 & Sleeps on Crate bed $(1 / 0)$ & 0.077 & 3.13 & 1.33 & 0.97 & 1.83 & Retain - Chi ${ }^{2}$ grp. D \\
\hline 16 & Sleeps on Non-carpeted floor + blankets $(1 / 0)$ & 0.248 & 1.34 & & & & Exclude \\
\hline 17 & Dog bed made of (comparator: soft + fleece/linen)* & 0.011 & 14.91 & & & & Exclude* \\
\hline & Soft cushion with waterproof cover & 0.767 & 0.09 & & & & Exclude* \\
\hline & Foam with fleece/linen cover & 0.033 & 4.53 & 1.37 & 1.03 & 1.83 & Exclude* \\
\hline & Foam with waterproof cover & 0.021 & 5.35 & 1.91 & 1.10 & 3.31 & Exclude* \\
\hline & Hypoallergenic material & 0.122 & 2.40 & 1.28 & 0.94 & 1.74 & Exclude* \\
\hline & Other & 0.083 & 3.01 & 0.65 & 0.40 & 1.06 & Exclude* \\
\hline 18 & Bed near heat source $(1 / 0)$ & 0.012 & 6.36 & 0.79 & 0.65 & 0.95 & Retain - Chi² grp. D \\
\hline 19 & Currently lives rural or urban (1-10 scale) & 0.153 & 2.04 & 1.03 & 0.99 & 1.06 & Exclude \\
\hline
\end{tabular}


(C) 2019. This accepted manuscript version is made available under the CC-BY-NC-ND 4.0 license http://creativecommons.org/licenses/by-nc-nd/4.0/

Table 2. Significant factors remaining in the final multivariate model with Case (1) or Control (0) as the outcome variable. Breed, although not significant, was forced into the model as a control.

\begin{tabular}{|c|c|c|c|c|c|c|}
\hline Factors & $\mathbf{p}$ & Wald & OR & $\begin{array}{c}\text { Lower } \\
\text { C.I }\end{array}$ & $\begin{array}{c}\text { Upper } \\
\text { C.I }\end{array}$ & Interpretation \\
\hline Labrador & 0.107 & 2.60 & & & & \\
\hline Male & $<0.001$ & 25.08 & 1.62 & 1.34 & 1.96 & Males more likely to be cases \\
\hline Flea/tick control given & $<0.001$ & 13.39 & 1.92 & 1.35 & 2.71 & Cases more likely to receive flea/tick control \\
\hline Neutered & $<0.001$ & 34.20 & 1.87 & 1.51 & 2.30 & Cases more likely to be neutered \\
\hline Born rural (vs. urban) & 0.013 & 6.10 & 0.74 & 0.58 & 0.94 & $\begin{array}{l}\text { Dogs born in rural areas less likely to be } \\
\text { cases }\end{array}$ \\
\hline Lives with other dogs & 0.001 & 10.45 & 0.73 & 0.61 & 0.89 & $\begin{array}{l}\text { Dogs that live with other } \\
\text { to be cases }\end{array}$ \\
\hline $\begin{array}{l}\text { Walked woods, fields } \\
\text { or beaches }\end{array}$ & $<0.001$ & 21.21 & 0.48 & 0.35 & 0.66 & $\begin{array}{l}\text { Dogs walked in woodlands, fields or } \\
\text { beaches are less likely to be cases }\end{array}$ \\
\hline $\begin{array}{l}\text { Spends time on } \\
\text { upholstery }\end{array}$ & 0.001 & 10.92 & 1.41 & 1.15 & 1.72 & $\begin{array}{l}\text { Dogs allowed on upholstered furniture are } \\
\text { more likely to be cases }\end{array}$ \\
\hline
\end{tabular}

Cox \& Snell R Square $=0.065$ 